\title{
Density of a gas of spin-polarized fermions in a magnetic field
}

\author{
S. Foulon, F. Brosens, and J. T. Devreese* \\ Departement Natuurkunde, Universiteit Antwerpen (UIA), Universiteitsplein 1, B-2610 Antwerpen, Belgium \\ L. F. Lemmens \\ Departement Natuurkunde, Universiteit Antwerpen (RUCA), Groenenborgerlaan 171, B-2020 Antwerpen, Belgium
}

(Received 23 July 2000; published 21 December 2000)

\begin{abstract}
For a fermion gas with equally spaced energy levels that is subjected to a magnetic field, the particle density is calculated. The derivation is based on the path integral approach for identical particles, in combination with the inversion techniques for the generating function of the static response functions. Explicit results are presented for the ground state density as a function of the magnetic field with a number of particles ranging from 1 to 45 .
\end{abstract}

DOI: 10.1103/PhysRevE.63.016111

PACS number(s): 05.30.-d, 03.75.Fi, 32.80.Pj

\section{INTRODUCTION}

The explosive growth of mesoscopic physics has made it possible to obtain a tunable number of electrons confined in semiconductor quantum dots [1]. Experiments on vertical quantum dots indicated that the confinement potential of single quantum dots is well described by a parabolic potential [2]. Although the electronic eigenstates and eigenvalues are essentially given by the Fock-Darwin states [3], detailed experimental data [4-6] reveal the importance of correlation effects on the ground state properties of the electrons in quantum dots. In order to take these correlation effects into account, various approximate theoretical methods [7-9] have been used, e.g., the eigenstates and eigenvalues of a harmonic interaction model including the effects of a magnetic field have been studied with operator techniques [10], and the harmonic interaction model has been used to explain specific features in the addition spectrum of a quantum dot in a magnetic field [11].

The harmonic interaction model is one of the rare examples for which the thermodynamical properties are exactly soluble, including the boson or fermion statistics, in the presence of a magnetic field [12]. It can also function as a trial model for the variational treatment of systems with more realistic interactions with the aid of the Jensen-Feynman inequality [13]. An example of this approach can be found in Ref. [14], where the Jensen-Feynman variational approach is used to describe Bose-Einstein condensation in a gas of ${ }^{87} \mathrm{Rb}$ and in a gas of ${ }^{7} \mathrm{Li}$ atoms. The spin statistics of the harmonic interaction model [15] can be treated within the same manybody path integral formalism.

In the present paper, we study the density of harmonically interacting electrons in a parabolic quantum dot in a magnetic field, taking into account the electron correlation effects analytically. This analysis is a natural extension of our previous investigation of the thermodynamical properties of a confined system of spin-polarized fermions in the presence of a magnetic field [12], using a method that combines the

\footnotetext{
*Also at Universiteit Antwerpen (RUCA) and at Technische Universiteit Eindhoven, NL 5600 MB Eindhoven, The Netherlands.
}

path integral formalism [16], the method of symmetrized density matrices [13], and inversion techniques for generating functions [17-19]. Instead of using the stochastic approach [20] with the Itô condition on the magnetic field [21] for calculating the path integral for $N$ identical interacting oscillators in a magnetic field, we relied on a detailed investigation of the classical equations of motion. The quantum mechanical corrections to their classical action are exactly taken into account.

The model system of $N$ harmonically interacting oscillators in a magnetic field is described by the Lagrangian (units with $\hbar$ and $m$ equal to unity are used throughout this paper)

$$
L=\frac{1}{2} \sum_{j=1}^{N}\left(\dot{\mathbf{r}}_{j}^{2}-2 \omega_{c} x_{j} \dot{y}_{j}\right)-V,
$$

where $\omega_{c}$ is the cyclotron frequency, and where the potential energy $V$ results from a harmonic confinement potential and a harmonic two-body interaction,

$$
V=\frac{\Omega^{2}}{2} \sum_{j=1}^{N} \mathbf{r}_{j}^{2} \pm \frac{\omega^{2}}{4} \sum_{j, l=1}^{N}\left(\mathbf{r}_{j}-\mathbf{r}_{l}\right)^{2}
$$

The two-body potential might be either attractive or repulsive depending on the plus sign or the minus sign in the two-body interaction.

As a first step in the calculation of the path integral, we studied the case of distinguishable particles [12]. Because the magnetic field affects the equations of motion only in the $x y$ plane perpendicular to the magnetic field, the Lagrangian naturally decouples into two contributions $L=L_{x y}+L_{z}$, where $L_{x y}$ contains the magnetic field. This allows us to calculate the propagator in the $x y$ plane independently from the propagator in the $z$ direction. Through a transformation to the center of mass reference frame, one obtains a set of $N$ three-dimensional oscillators in a magnetic field. The center of mass is described by an oscillator with frequency $\Omega$. The remaining $N-1$ oscillators, associated with the internal degrees of freedom, have a frequency

$$
w=\sqrt{\Omega^{2} \pm N \omega^{2}}
$$


Note that the case of a repulsive interaction imposes a stability constraint on the confinement potential: $\Omega$ has to be large enough to keep the repelling particles together. The magnetic field does not affect these frequencies in the $z$ direction. But in the $x y$ plane the equations of motion reveal two renormalized frequencies due to the magnetic field,

$$
s=\sqrt{w^{2}+\omega_{L}^{2}} \quad \text { and } \quad s_{c . m .}=\sqrt{\Omega^{2}+\omega_{L}^{2}},
$$

where $\omega_{L}=\omega_{c} / 2$ denotes the Larmor frequency. The index c.m. refers to the renormalized frequency in the center of mass coordinates. Once the propagator for distinguishable particles is known, it is projected on the antisymmetric representation to obtain the fermion propagator [12].

This paper is organized as follows. In Sec. II the oneparticle correlation function is calculated for identical harmonically interacting oscillators in a homogeneous magnetic field. In Sec. III special attention is paid to the fermion ground state density in the $x y$ and $x z$ planes. In Sec. IV some concluding remarks are given.

\section{STATIC RESPONSE PROPERTIES OF THE MODEL SYSTEM}

In the path integral approach to quantum mechanics the expectation value of an expression $A(\overline{\mathbf{r}})$ is given by

$$
\langle A(\overline{\mathbf{r}}, \beta)\rangle_{I}=\frac{\int d \mathbf{r}_{1} \cdots \int d \mathbf{r}_{N} K_{I}(\overline{\mathbf{r}}, \beta \mid \overline{\mathbf{r}}, 0) A(\overline{\mathbf{r}})}{\int d \mathbf{r}_{1} \cdots \int d \mathbf{r}_{N} K_{I}(\overline{\mathbf{r}}, \beta \mid \overline{\mathbf{r}}, 0)},
$$

where $\overline{\mathbf{r}}$ is the $3 N$-dimensional vector containing the coordinates $\mathbf{r}_{1}, \ldots, \mathbf{r}_{N}$ of all the $N$ particles, and $K_{I}\left(\overline{\mathbf{r}}, \beta \mid \overline{\mathbf{r}}^{\prime}, 0\right)$ denotes the propagator in the Euclidean time $\beta=1 /\left(k_{B} T\right)$, with $k_{B}$ denoting the Boltzmann constant and $T$ the temperature. The subscript $I$ emphasizes that identical particles (fermions or bosons) are considered. For the probability density and its Fourier transform this gives

$$
\begin{aligned}
n(\mathbf{r}) & =\frac{1}{N}\left\langle\sum_{l=1}^{N} \delta\left(\mathbf{r}-\mathbf{r}_{l}\right)\right\rangle_{I} \\
& =\int \frac{d \mathbf{q}}{(2 \pi)^{3}} n_{\mathbf{q}} e^{-i \mathbf{q} \cdot \mathbf{r}^{\leftrightarrow}} n_{\mathbf{q}} \\
& =\frac{1}{N} \sum_{l=1}^{N}\left\langle e^{i \mathbf{q} \cdot \mathbf{r}_{l}}\right\rangle_{I} .
\end{aligned}
$$

Substituting the expression for the propagator $K_{I}(\overline{\mathbf{r}}, \beta \mid \overline{\mathbf{r}}, 0)$, one obtains the following integral for the Fourier transform $n_{\mathbf{q}}$ :

$$
\begin{aligned}
n_{\mathbf{q}}= & \frac{1}{N Z_{I}(\beta, N)} \iint \frac{d \mathbf{R} d \mathbf{k}}{(2 \pi)^{3}} e^{i \mathbf{k} \cdot \mathbf{R}} \frac{K_{\Omega}(\sqrt{N} Z, \beta \mid \sqrt{N} Z, 0) K_{\omega_{L}, s_{c . m} .}(\sqrt{N} X, \sqrt{N} Y, \beta \mid \sqrt{N} X, \sqrt{N} Y, 0)}{K_{w}(\sqrt{N} Z, \beta \mid \sqrt{N} Z, 0) K_{\omega_{L}, s}(\sqrt{N} X, \sqrt{N} Y, \beta \mid \sqrt{N} X, \sqrt{N} Y, 0)} \\
& \times \int d \overline{\mathbf{r}} e^{-i \overline{\mathbf{k}} \cdot \overline{\mathbf{r}}} \sum_{l} e^{i \mathbf{q} \cdot \mathbf{r}_{l}} \frac{1}{N !} \sum_{p} \xi^{p} \prod_{j=1}^{N} K_{w}\left((P z)_{j}, \beta \mid z_{j}, 0\right) K_{\omega_{L}, s}\left((P x)_{j},(P y)_{j}, \beta \mid x_{j}, y_{j}, 0\right),
\end{aligned}
$$

where $Z_{I}(\beta, N)=\int d \overline{\mathbf{r}} K_{I}(\overline{\mathbf{r}}, \beta \mid \overline{\mathbf{r}}, 0)$ is the partition function of $N$ identical particles, $P$ denotes a permutation of the particle indices, and $\xi=+1$ for bosons or -1 for fermions. The propagators $K_{\Omega}, K_{w}, K_{\omega_{L}, s_{c . m .}}$, and $K_{\omega_{L}, s}$ are known in closed form, and explicitly calculated in Ref. [12]. In order to obtain tractable expressions for $n_{\mathbf{q}}$, the summation over all possible permutations will be rewritten as a sum over all possible cycles.

\section{One-particle expectation values}

For the one-point correlation function a factor $e^{i \mathbf{q} \cdot \mathbf{r}_{l}}$ has to be taken into account in each permutation when applying the cyclic decomposition of the permutations. Indicating the number of cycles of length $\ell$ by $M_{\ell}$, the cyclic decomposition for $n_{\mathbf{q}}$ becomes

$$
\begin{aligned}
& n_{\mathbf{q}}=\frac{1}{N Z_{I}(\beta, N)} \iint \frac{d \mathbf{R} d \mathbf{k}}{(2 \pi)^{3}} e^{i \mathbf{k} \cdot \mathbf{R}} \frac{K_{\Omega}(\sqrt{N} Z, \beta \mid \sqrt{N} Z, 0) K_{\omega_{L}, s_{c, m} .}(\sqrt{N} X, \sqrt{N} Y, \beta \mid \sqrt{N} X, \sqrt{N} Y, 0)}{K_{w}(\sqrt{N} Z, \beta \mid \sqrt{N} Z, 0) K_{\omega_{L}, s}(\sqrt{N} X, \sqrt{N} Y, \beta \mid \sqrt{N} X, \sqrt{N} Y, 0)}
\end{aligned}
$$

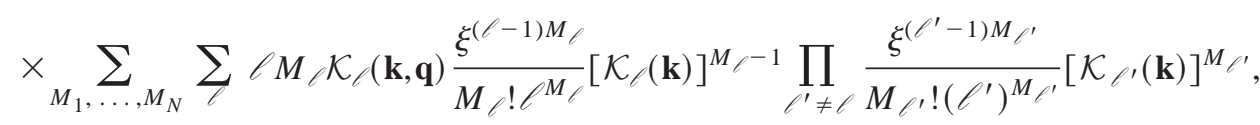

where 


$$
\mathcal{K}_{\ell}(\mathbf{k}, \mathbf{q})=\int d \mathbf{r}_{\ell+1} \cdots \int d \mathbf{r}_{1} \delta\left(\mathbf{r}_{\ell+1}-\mathbf{r}_{1}\right) e^{i \mathbf{q} \cdot \mathbf{r}_{1}} \prod_{j=1}^{\ell} K_{\omega_{L, s}}\left(x_{j+1}, y_{j+1}, \beta \mid x_{j}, y_{j}, 0\right) K_{w}\left(z_{j+1}, \beta \mid z_{j}, 0\right) e^{-i \mathbf{k} \cdot \mathbf{r}_{j} / N},
$$

and $\mathcal{K}_{\ell}(\mathbf{k}, \mathbf{q}=0)=\mathcal{K}_{\curlywedge}(\mathbf{k})$ is the same function as Eq. (17) of Ref. [12] in the calculation of the partition function $Z_{I}(\beta, N)$. We point out that the positive integers $\ell$ and $M_{\ell}$ (with $1 \leqslant \ell \leqslant N$ ) have to satisfy the constraint $\Sigma \ell M_{\ell}=N$. Taking into account the semigroup property of the propagators $K_{\omega_{L}, s}\left(x_{j+1}, y_{j+1}, \beta \mid x_{j}, y_{j}, 0\right)$ and $K_{w}\left(z_{j+1}, \beta \mid z_{j}, 0\right)$, one recognizes in $\mathcal{K} /(\mathbf{k}, \mathbf{q})$ the partition function (over a time interval $\ell \beta$ ) of a driven harmonic oscillator in a magnetic field,

$$
\mathcal{K}(\mathbf{k}, \mathbf{q})=\iiint K_{\omega_{L}, s}(x, y, \ell \beta \mid x, y, 0) K_{w}(z, \ell \beta \mid z, 0) \exp \left(-\int_{0}^{\ell \beta} \mathbf{f}_{\mathbf{q}}(\tau) \mathbf{r}(\tau) d \tau\right) d x d y d z,
$$

with the driving force

$$
\mathbf{f}_{\mathbf{q}}(\tau)=i \frac{\mathbf{k}}{N} \sum_{j=0}^{\ell-1} \delta(\tau-j \beta)-i \mathbf{q} \delta(\tau)
$$

This partition function is known in closed form and given by

$$
\mathcal{K}_{\ell}(\mathbf{k}, \mathbf{q})=\frac{1}{2\left(\cosh \ell \beta s-\cosh \ell \beta \omega_{L}\right)} \exp \left(\frac{\phi_{q_{x}, q_{y}}}{4 s\left(\cosh \ell \beta s-\cosh \ell \beta \omega_{L}\right)}\right)\left(\frac{1}{2 \sinh (\ell \beta w / 2)} e^{\phi_{q_{z}}}\right),
$$

with

$$
\begin{aligned}
& \phi_{q_{x}, q_{y}}= \int_{0}^{\ell \beta} \int_{0}^{\ell \beta}\left[f_{q_{x}}(\tau) f_{q_{x}}(\sigma)+f_{q_{y}}(\tau) f_{q_{y}}(\sigma)\right]\left[\cosh \omega_{L}(\tau-\sigma) \sinh s(\ell \beta-|\tau-\sigma|)\right. \\
&\left.+\cosh \omega_{L}(\ell \beta-|\tau-\sigma|) \sinh s|\tau-\sigma|\right] d \sigma d \tau+i \int_{0}^{\ell \beta} \int_{0}^{\ell \beta}\left[f_{q_{x}}(\tau) f_{q_{y}}(\sigma)-f_{q_{x}}(\sigma) f_{q_{y}}(\tau)\right] \\
& \times\left[\sinh \omega_{L}(\tau-\sigma) \sinh s(\ell \beta-|\sigma-\tau|)-\sinh \omega_{L}(\ell \beta-|\sigma-\tau|) \sinh s(\tau-\sigma)\right] d \sigma d \tau, \\
& \phi_{q_{z}}=\frac{1}{2} \int_{0}^{\ell \beta} \int_{0}^{\ell \beta} \frac{f_{q_{z}}(\tau) f_{q_{z}}(\sigma)}{2 w} \frac{\cosh (\ell \beta / 2-|\tau-\sigma| w)}{\sinh \ell \beta w / 2} d \sigma d \tau .
\end{aligned}
$$

Substituting the force $\mathbf{f}_{\mathbf{q}}(\tau)$ from Eq. (11) into the above expressions for $\phi_{q_{x}, q_{y}}$ and $\phi_{q_{z}}$ yields

$$
\begin{aligned}
\mathcal{K}_{\curlywedge}(\mathbf{k}, \mathbf{q})= & \mathcal{K}_{\curlywedge}\left(k_{x}, k_{y}\right) \exp \left(\frac{\left(k_{x} q_{x}+k_{y} q_{y}\right) \sinh \beta s}{2 N s\left(\cosh \beta s-\cosh \beta \omega_{L}\right)}-\frac{\left(q_{x}^{2}+q_{y}^{2}\right) \sinh \ell \beta s}{4 s\left(\cosh \ell \beta s-\cosh \ell \beta \omega_{L}\right)}\right) \\
& \times \mathcal{K}_{\curlywedge}\left(k_{z}\right) \exp \left(\frac{k_{z} q_{z}}{2 N w} \operatorname{coth} \frac{1}{2} \beta w-\frac{q_{z}^{2}}{4 w} \operatorname{coth} \frac{1}{2} \ell \beta w\right),
\end{aligned}
$$

with

$$
\begin{gathered}
\mathcal{K}_{\ell}\left(k_{x}, k_{y}\right)=\frac{1}{2\left(\cosh \ell \beta s-\cosh \ell \beta \omega_{L}\right)} \exp \left(-\frac{\ell\left(k_{x}^{2}+k_{y}^{2}\right)}{4 N^{2} s} \frac{\sinh \beta s}{\cosh \beta s-\cosh \beta \omega_{L}}\right), \\
\mathcal{K}_{\ell}\left(k_{z}\right)=\frac{1}{2(\cosh \ell \beta w-1)} \exp \left(-\frac{\ell k_{z}^{2}}{4 N^{2} w} \operatorname{coth} \frac{\beta w}{2}\right) .
\end{gathered}
$$

The remaining integrations over $\mathbf{k}$ and $\mathbf{R}$ in $n_{\mathbf{q}}$ are Gaussian and easy to perform, eventually leading to

$$
n_{\mathbf{q}}=\exp \left[-\frac{\left(q_{x}^{2}+q_{y}^{2}\right)}{4 N}\left(\frac{\sinh \beta s_{c . m .}}{s_{c . m .}\left(\cosh \beta s_{c . m .}-\cosh \beta \omega_{L}\right)}-\frac{\sinh \beta s}{s\left(\cosh \beta s-\cosh \beta \omega_{L}\right)}\right)-\frac{q_{z}^{2}}{4 N}\left(\frac{\operatorname{coth} \beta \Omega / 2}{\Omega}-\frac{\operatorname{coth} \beta w / 2}{w}\right)\right] \tilde{n}_{\mathbf{q}},
$$


with

$$
\begin{aligned}
\tilde{n}_{\mathbf{q}}= & \frac{1}{N Z_{I}(\beta, N)} \sum_{M_{1}, \ldots, M_{N}}\left[\sum \ell M_{\ell} \exp \left(-\frac{\left(q_{x}^{2}+q_{y}^{2}\right) \sinh \ell \beta s}{4 s\left(\cosh \ell \beta s-\cosh \ell \beta \omega_{L}\right)}-\frac{q_{z}^{2}}{4 w} \operatorname{coth} \frac{1}{2} \ell \beta w\right)\right] \\
& \times \prod \frac{\xi^{(\ell-1) M_{\ell}}}{M_{\ell} ! \ell^{M_{\ell}}}\left(\frac{1}{4\left(\cosh \ell \beta s-\cosh \ell \beta \omega_{L}\right) \sinh \frac{1}{2} \ell \beta w}\right)^{M_{\ell}} .
\end{aligned}
$$

The exponential factor in $n_{\mathbf{q}}$ accounts for the center of mass contribution, and it becomes unity for noninteracting particles $(w=\Omega)$. The factor $\tilde{n}_{\mathbf{q}}$ is the expectation value of $\Sigma_{l} e^{i \mathbf{q} \cdot \mathbf{r}_{l}}$ in the subspace of the relative coordinate system with its corresponding partition function $\mathbb{Z}_{I}(\beta, N)$.

We now introduce the generating function $\mathcal{G}_{1}(\beta, u, \mathbf{q})=\sum_{N=0}^{\infty}\left[N Z_{I}(\beta, N) \tilde{n}_{\mathbf{q}}\right] u^{N}$ for the Fourier transform of the density, as was done before $[17,18]$ in the absence of a magnetic field,

$\mathcal{G}_{1}(\beta, u, \mathbf{q})$

$$
\begin{aligned}
= & \sum_{N=0}^{\infty} \sum_{M_{1}, \ldots, M_{N}}\left[\sum \ell M_{\ell} \exp \left(-\frac{\left(q_{x}^{2}+q_{y}^{2}\right) \sinh \ell \beta s}{8 s \sinh (\ell \beta / 2)\left(s+\omega_{L}\right) \sinh (\ell \beta / 2)\left(s-\omega_{L}\right)}-\frac{q_{z}^{2}}{4 w} \operatorname{coth} \frac{1}{2} \ell \beta w\right)\right] \\
& \times \prod \frac{1}{M_{\ell} !}\left(\frac{\xi^{(\ell-1)} u^{\ell}}{\ell\left(8 \sinh (\ell \beta / 2)\left(s+\omega_{L}\right) \sinh (\ell \beta / 2)\left(s-\omega_{L}\right) \sinh \frac{1}{2} \ell \beta w\right)}\right)^{M_{\ell}} \\
= & \Xi_{I}(\beta, u) \sum_{\ell=1}^{\infty} \frac{\xi^{(\ell-1)} \exp \left\{-\left[\left(q_{x}^{2}+q_{y}^{2}\right) \sinh \ell \beta s / 8 s \sinh (\ell \beta / 2)\left(s+\omega_{L}\right) \sinh (\ell \beta / 2)\left(s-\omega_{L}\right)\right]-\left(q_{z}^{2} / 4 w\right) \operatorname{coth} \frac{1}{2} \ell \beta w\right\}}{8 \sinh (\ell \beta / 2)\left(s+\omega_{L}\right) \sinh (\ell \beta / 2)\left(s-\omega_{L}\right) \sinh \frac{1}{2} \ell \beta w} u^{\ell},
\end{aligned}
$$

where $\Xi_{I}(\beta, u)=\sum_{N=0}^{\infty} \mathbb{Z}_{I}(\beta, N) u^{N}$ is the generating function for the partition function $\mathbb{Z}_{I}(\beta, N)$. After straightforward algebra one is left with

$$
\begin{aligned}
\tilde{n}_{\mathbf{q}}= & \frac{1}{N} \sum_{\ell=1}^{N} \xi^{(\ell-1)} \frac{Z_{I}(\beta, N-\ell)}{Z_{I}(\beta, N)} \\
& \times \frac{\exp \left\{-\left[\left(q_{x}^{2}+q_{y}^{2}\right) \sinh \ell \beta s / 8 s \sinh (\ell \beta / 2)\left(s+\omega_{L}\right) \sinh (\ell \beta / 2)\left(s-\omega_{L}\right)\right]-\left(q_{z}^{2} / 4 w\right) \operatorname{coth} \frac{1}{2} \ell \beta w\right\}}{8 \sinh (\ell \beta / 2)\left(s+\omega_{L}\right) \sinh (\ell \beta / 2)\left(s-\omega_{L}\right) \sinh \frac{1}{2} \ell \beta w} .
\end{aligned}
$$

It is noted that in the limit $\mathbf{q} \rightarrow 0$ the sum rule $\tilde{n}_{\mathbf{q}=0}=1$ is indeed satisfied. The density $n(\mathbf{r})$ in real space then becomes

$$
\begin{aligned}
n(\mathbf{r}) & =\int \frac{d \mathbf{q}}{(2 \pi)^{3}} n_{\mathbf{q}} e^{-i \mathbf{q} \cdot \mathbf{r}} \\
& =\frac{1}{N} \sum_{\ell=1}^{N} \xi^{(\ell-1)} \frac{Z_{I}(\beta, N-\ell)}{\mathbb{Z}_{I}(\beta, N)} \frac{s \mathcal{B}_{\ell}}{\pi} \sqrt{\frac{w \mathcal{A} \ell}{\pi}} \frac{\exp \left[-s \mathcal{B} /\left(x^{2}+y^{2}\right)-w \mathcal{A} / z^{2}\right]}{8 \sinh (\ell \beta / 2)\left(s+\omega_{L}\right) \sinh (\ell \beta / 2)\left(s-\omega_{L}\right) \sinh \frac{1}{2} \ell \beta w},
\end{aligned}
$$

with

$$
\begin{gathered}
\mathcal{A}_{\ell}=\left[\operatorname{coth} \frac{1}{2} \ell \beta w+\frac{1}{N}\left(\frac{w}{\Omega} \operatorname{coth} \frac{\beta \Omega}{2}-\operatorname{coth} \frac{\beta w}{2}\right)\right]^{-1}, \\
\mathcal{B}_{\ell}=\left[\frac{\sinh \ell \beta s}{\cosh \ell \beta s-\cosh \ell \beta \omega_{L}}+\frac{1}{N}\left(\frac{s}{s_{c . m .}} \frac{\sinh \beta s_{c . m .}}{\cosh \beta s_{c . m .}-\cosh \beta \omega_{L}}-\frac{\sinh \beta s}{\cosh \beta s-\cosh \beta \omega_{L}}\right)\right]^{-1} .
\end{gathered}
$$

The sum rule $\int d \mathbf{r} n(\mathbf{r})=1$ for the density is easily verified. In the next subsection the fermion ground state density will be examined, by inverting the defining series for the generating function $\mathcal{G}_{1}(\beta, u, \mathbf{q})$. Subsequently results will be presented for the ground state density in the $x y$ plane and in the $x z$ plane.

\section{GROUND STATE DENSITY}

Because of alternating signs in the recurrence relations for the partition functions $Z_{I}(\beta, N)$, Eq. (22) is not appropriate for numerical purposes, in particular in the low temperature limit. This sign problem can be circumvented by an alternative inversion of the generating function $\mathcal{G}_{1}$ using contour integration: 


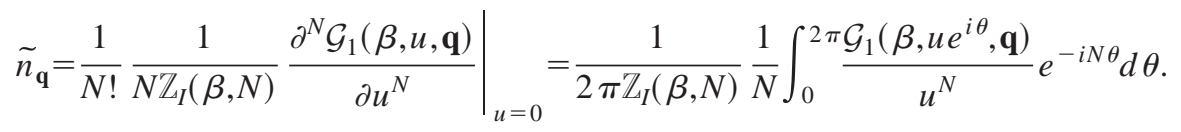

Substituting Eq. (19) into the above expression yields

$$
\begin{aligned}
\tilde{n}_{\mathbf{q}}= & \frac{1}{N} \frac{\Xi_{I}(\beta, u) / u^{N}}{2 \pi Z_{I}(\beta, N)} e^{-\left(\kappa_{x}^{2}+\kappa_{y}^{2}+\kappa_{z}^{2}\right)} \\
& \times \sum_{\ell=1}^{\infty} \int_{0}^{2 \pi} \frac{\Xi_{I}\left(\beta, u e^{i \theta}\right)}{\Xi_{I}(\beta, u)} \frac{\xi^{(\ell-1)}\left(\sqrt{b b_{1} b_{2}} u e^{i \theta}\right)^{\ell} \exp \left\{-\left(\kappa_{x}^{2}+\kappa_{y}^{2}\right)\left[b_{1}^{\ell} /\left(1-b_{1}^{\ell}\right)+b_{2}^{\ell} /\left(1-b_{2}^{\ell}\right)\right]\right\} \exp \left[-2 \kappa_{z}^{2} b^{\ell} /\left(1-b^{\prime}\right)\right]}{\left(1-b^{\ell}\right)\left(1-b_{1}^{\prime}\right)\left(1-b_{2}^{\prime}\right)} \\
& \times e^{-i N \theta} d \theta
\end{aligned}
$$

with the shorthand notations

$$
\kappa_{x}^{2}=q_{x}^{2} / 4 s, \quad \kappa_{y}^{2}=q_{y}^{2} / 4 s, \quad \kappa_{z}^{2}=q_{z}^{2} / 4 w, \quad b=e^{-\beta w}, \quad b_{1}=e^{-\beta\left(s+\omega_{L}\right)}, \quad b_{2}=e^{-\beta\left(s-\omega_{L}\right)} .
$$

By expanding the Fourier transform of the density $\tilde{n}_{\mathbf{q}}$ in powers of $b, b_{1}$, and $b_{2}$ one arrives at

$$
\begin{aligned}
& \tilde{n}_{\mathbf{q}}=\frac{1}{N} \frac{\Xi_{I}(\beta, u)}{2 \pi Z_{I}(\beta, N) u^{N}} e^{-\left(\kappa_{x}^{2}+\kappa_{y}^{2}+\kappa_{z}^{2}\right)} \sum_{m=0}^{\infty} \sum_{m_{1}=0}^{\infty} \sum_{n_{1}=0}^{\infty} \sum_{m_{2}=0}^{\infty} \sum_{n_{2}=0}^{\infty} \frac{\left(-\kappa_{x}^{2}\right)^{m_{1}+m_{2}}\left(-\kappa_{y}^{2}\right)^{n_{1}+n_{2}}\left(-2 \kappa_{z}^{2}\right)^{m}}{\Gamma\left(m_{1}+1\right) \Gamma\left(m_{2}+1\right) \Gamma\left(n_{1}+1\right) \Gamma\left(n_{2}+1\right) \Gamma(m+1)} \\
& \times \sum_{k=0}^{\infty} \sum_{k_{1}=0}^{\infty} \sum_{k_{2}=0}^{\infty} \frac{\left(m_{1}+n_{1}+1\right)_{k_{1}}\left(m_{2}+n_{2}+1\right)_{k_{2}}(m+1)_{k}}{\Gamma\left(k_{1}+1\right) \Gamma\left(k_{2}+1\right) \Gamma(k+1)}
\end{aligned}
$$

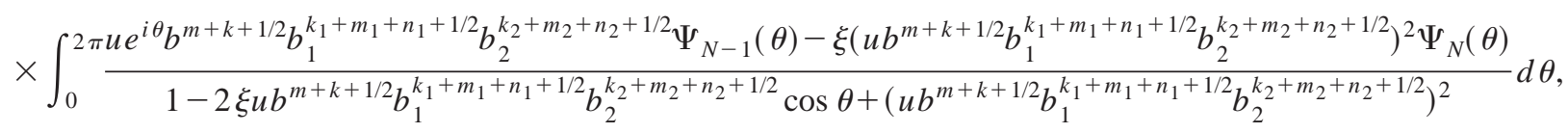

with $(a)_{p}=\Gamma(a+p) / \Gamma(a)$ the Pochhammer symbol. The function $\Psi_{N}(\theta)=e^{-i N \theta} \Xi_{I}\left(\beta, u e^{i \theta}\right) / \Xi_{I}(\beta, u)$ has previously been obtained [12]. Using Eq. (6), the density (still at arbitrary temperature) then becomes

$$
\begin{aligned}
& n(\mathbf{r})=\frac{1}{N} \frac{1}{\int_{0}^{2 \pi} \Psi_{N}(\theta) d \theta} \sqrt{\frac{s^{2} w}{\pi^{3} A^{2} B}} \exp \left(-\frac{s\left(x^{2}+y^{2}\right)}{A}-\frac{w z^{2}}{B}\right) \\
& \times \sum_{m=0}^{\infty} \sum_{m_{1}=0}^{\infty} \sum_{n_{1}=0}^{\infty} \sum_{m_{2}=0}^{\infty} \sum_{n_{2}=0}^{\infty} \frac{(-1 / 4 A)^{m_{1}+m_{2}+n_{1}+n_{2}}(-1 / 2 B)^{m}}{\Gamma\left(m_{1}+1\right) \Gamma\left(m_{2}+1\right) \Gamma\left(n_{1}+1\right) \Gamma\left(n_{2}+1\right) \Gamma(m+1)} \\
& \times \sum_{k=0}^{\infty} \sum_{k_{1}=0}^{\infty} \sum_{k_{2}=0}^{\infty} \frac{\left(m_{1}+n_{1}+1\right)_{k_{1}}\left(m_{2}+n_{2}+1\right)_{k_{2}}(m+1)_{k}}{\Gamma\left(k_{1}+1\right) \Gamma\left(k_{2}+1\right) \Gamma(k+1)} \\
& \times \sum_{q=0}^{m} \sum_{q_{1}=0}^{m_{1}+m_{2}} \sum_{q_{2}=0}^{n_{1}+n_{2}} \frac{\left(-4 s x^{2} / A\right)^{m_{1}+m_{2}-q_{1}}\left(-4 s y^{2} / A\right)^{n_{1}+n_{2}-q_{2}}\left(-4 w z^{2} / B\right)^{m-q}}{\left[2\left(m_{1}+m_{2}\right)+1\right]_{-2 q_{1}} \Gamma\left(q_{1}+1\right)\left[2\left(n_{1}+n_{2}\right)+1\right]_{-2 q_{2}} \Gamma\left(q_{2}+1\right)(2 m+1)_{-2 q} \Gamma(q+1)}
\end{aligned}
$$

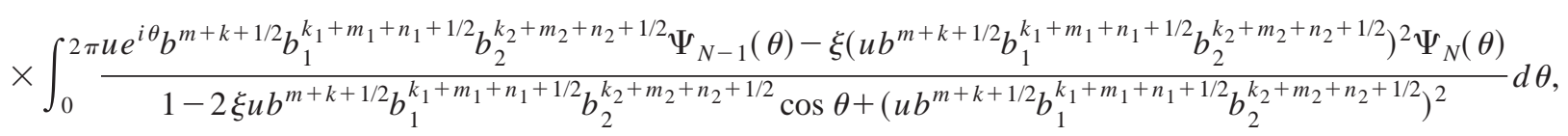

with

$$
\begin{gathered}
A=1+\frac{1}{N}\left(\frac{s}{s_{c . m .}} \frac{\sinh \beta s_{c . m .}}{\cosh \beta s_{c . m .}-\cosh \beta \omega_{L}}-\frac{\sinh \beta s}{\cosh \beta s-\cosh \beta \omega_{L}}\right), \\
B=1+\frac{1}{N}\left(\frac{w}{\Omega} \operatorname{coth} \frac{\beta \Omega}{2}-\operatorname{coth} \frac{\beta w}{2}\right) .
\end{gathered}
$$


The ground state density at $T=0$ is then readily obtained by taking the limit $\beta \rightarrow \infty$ :

$$
\begin{aligned}
& n_{T=0}(\mathbf{r})=\frac{1}{N} \sqrt{\frac{(\sigma s)^{2} \vartheta w}{\pi^{3}}} \exp \left[-\sigma s\left(x^{2}+y^{2}\right)-\vartheta w z^{2}\right] \\
& \times \sum_{m=0}^{\infty} \sum_{m_{1}=0}^{\infty} \sum_{n_{1}=0}^{\infty} \sum_{m_{2}=0}^{\infty} \sum_{n_{2}=0}^{\infty} \frac{(-\sigma / 4)^{m_{1}+m_{2}+n_{1}+n_{2}}(-\vartheta / 2)^{m}}{\Gamma\left(m_{1}+1\right) \Gamma\left(m_{2}+1\right) \Gamma\left(n_{1}+1\right) \Gamma\left(n_{2}+1\right) \Gamma(m+1)} \\
& \times \sum_{k=0}^{\infty} \sum_{k_{1}=0}^{\infty} \sum_{k_{2}=0}^{\infty} \frac{\left(m_{1}+n_{1}+1\right)_{k_{1}}\left(m_{2}+n_{2}+1\right)_{k_{2}}(m+1)_{k}}{\Gamma\left(k_{1}+1\right) \Gamma\left(k_{2}+1\right) \Gamma(k+1)}
\end{aligned}
$$

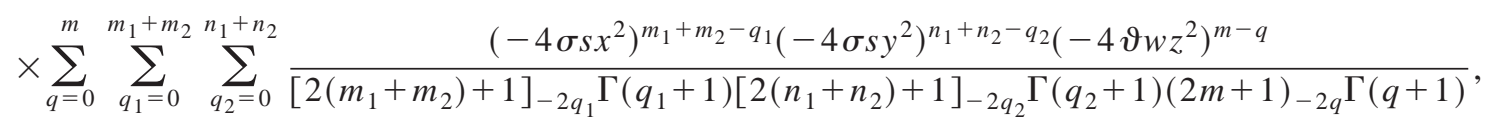

with

$$
\sigma=\frac{N}{N-1+s / s_{c . m .}} \quad \text { and } \quad \vartheta=\frac{N}{N-1+w / \Omega}
$$

The sum rule $\int d \mathbf{r} n_{T=0}(\mathbf{r})=1$ can easily be verified.

\section{A. Density in the $x y$ plane}

The ground state surface density $n(x, y)$ $\equiv n_{T=0}(x, y, z) / n_{T=0}(0,0, z)$ in the $x y$ plane is cylindrically symmetric, which means that $n(x=0, y)$ contains all the in-

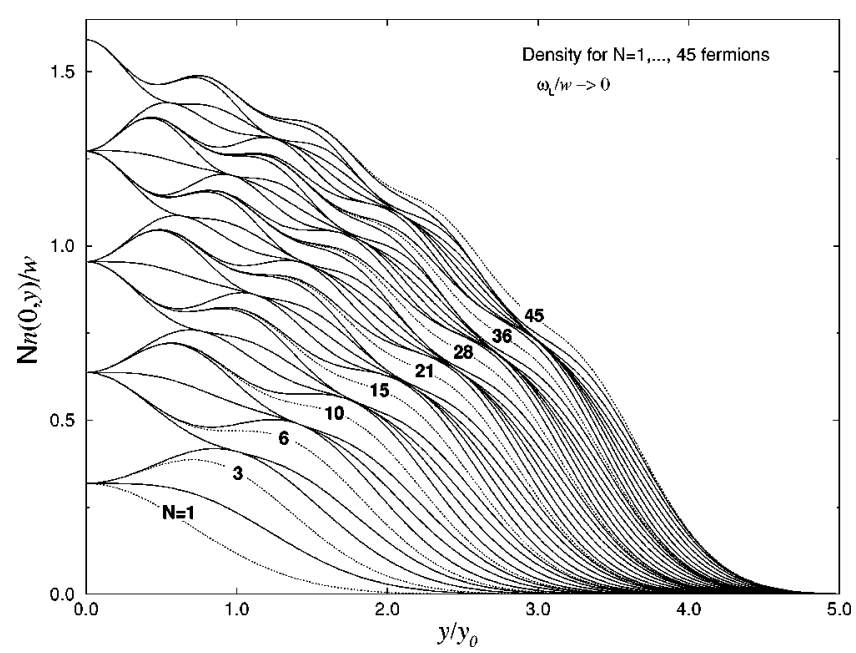

FIG. 1. Scaled surface density $N n(x=0, y) w$ for $\Omega=w$ in the plane perpendicular to the magnetic field, as a function of the scaled distance $y / y_{0}$ (with $y_{0}=1 / \sqrt{w}$; units with $\hbar=m=1$ are used) from the center of the parabolic confinement potential for $N=1, \ldots, 45$ fermions in the limit $\omega_{L} / w \rightarrow 0$. The densities corresponding to 1 , $3,6,10,15,21,28,36$, and 45 fermions (i.e., for closed shell configurations in the absence of a magnetic field) are indicated by dashed lines and the corresponding particle number is explicitly indicated.
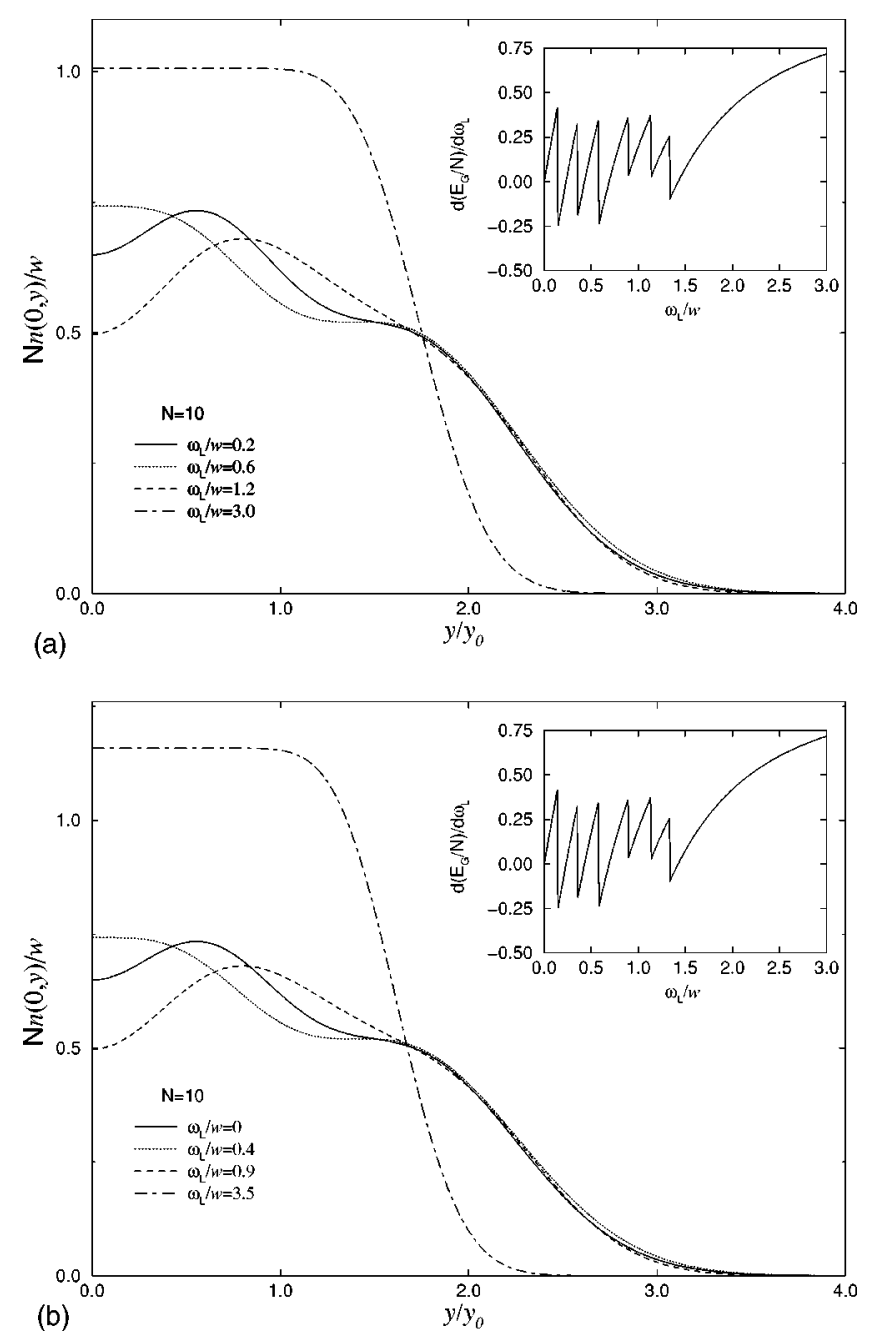

FIG. 2. (a) Scaled surface density $N n(x=0, y) / w$ for $\Omega=w$ in the plane perpendicular to the magnetic field, as a function of the scaled distance $y / y_{0}$ (with $y_{0}=1 / \sqrt{w}$ ) from the center of the parabolic confinement potential for $N=10$ fermions and for various Larmor frequencies $\omega_{L} / w=0.2,0.6,1.2,3.0$. The inset shows the magnetic susceptibility for $N=10$ fermions as a function of the Larmor frequency. (b) Same as in (a), but for $\omega_{L} / w=0,0.4,0.9$. 


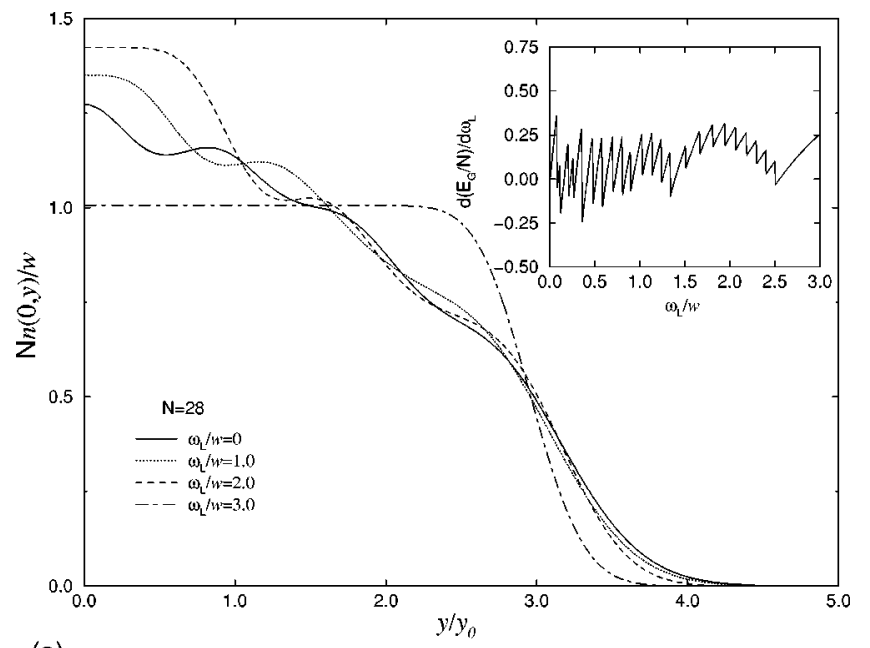

(a)

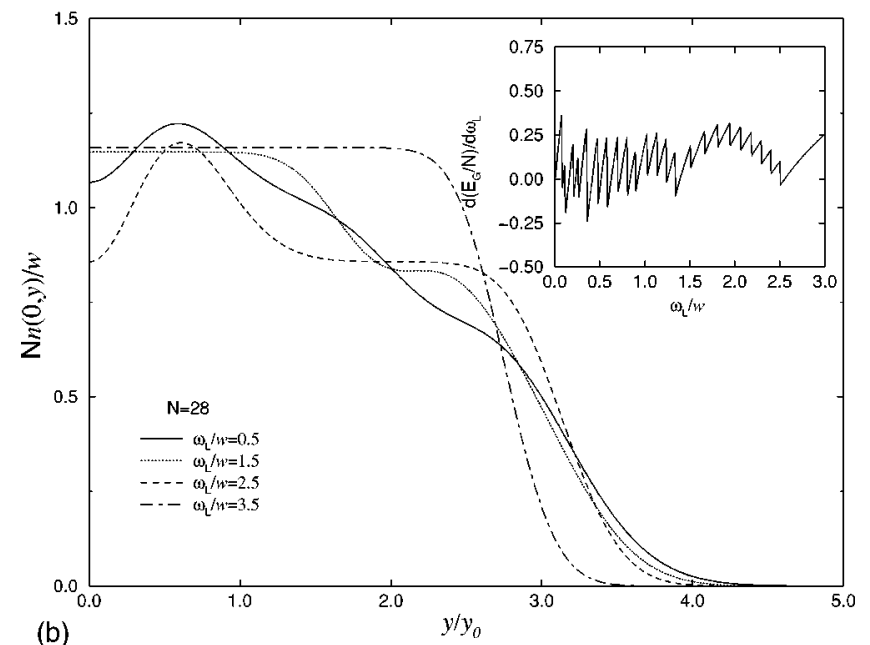

(b)

FIG. 3. (a) Scaled surface density $N n(x=0, y) / w$ for $\Omega=w$ as a function of the scaled distance $y / y_{0}$ (with $y_{0}=1 / \sqrt{w}$ ) from the center of the parabolic confinement potential for $N=28$ fermions and for various Larmor frequencies $\omega_{L} / w=0,1.0,2.0,3.0$. The inset shows the magnetic susceptibility for $N=28$ fermions as a function of $\omega_{L}$. (b) Same as in (a), but for $\omega_{L} / w=0.5,1.5,2.5,3.5$.

formation. Figure 1 shows $N n(x=0, y) / w$ for $1-45$ electrons for the noninteracting case $(w=\Omega)$ at zero temperature. The factor $1 / w$ makes this expression dimensionless (in the units with $\hbar=m=1$ used throughout this paper). The factor $N$ is introduced for clarity in the figure, in order to avoid too strongly overlapping curves. The densities for $N=1,3,6$, $10,15,21,28,36$, and 45 electrons, i.e., for closed shell configurations, are emphasized by dashed lines. Oscillations appear in the density profiles, indicating concentric orbitals of increased density around the center of the confinement potential. The oscillations become more pronounced if the number of particles increases. Previously [22] the effects of an attractive $(w>\Omega)$ and a repulsive $(w<\Omega)$ two-particle interaction were studied in the absence of a magnetic field. It was seen that a repulsive interaction induced an expansion whereas an attractive interaction induced a contraction of the gas of interacting fermions. These effects also appear for
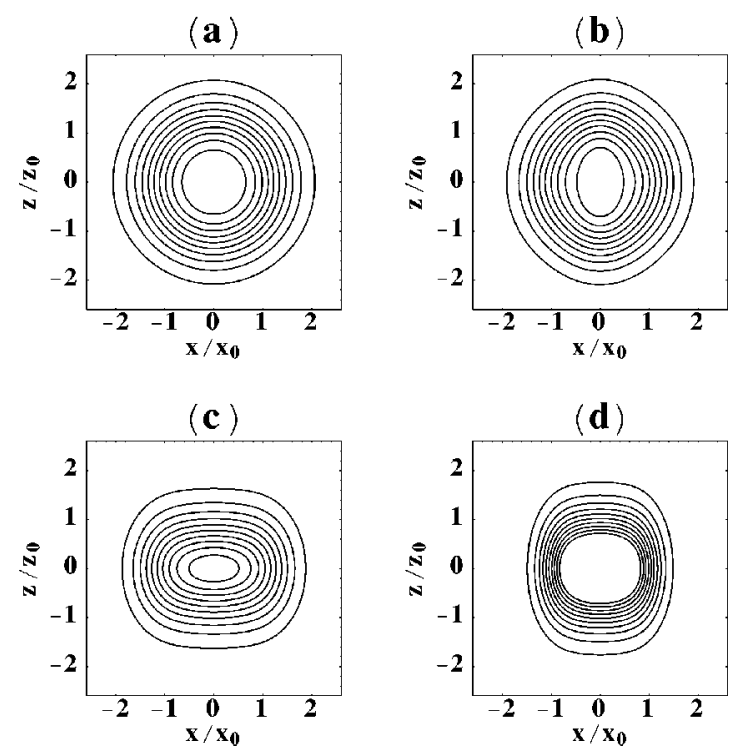

FIG. 4. Contour plots of $N n(x, z) / w$ for $N=4$ fermions and for $\Omega=w$ as a function of the scaled coordinates $x / x_{0}$ and $z / z_{0}$ with $x_{0}=z_{0}=1 / \sqrt{w}$, measured from the center of the parabolic confinement potential, for different values of the magnetic field: (a) $\omega_{L} / w=0.2$, (b) $\omega_{L} / w=1.0$, (c) $\omega_{L} / w=1.5$, and (d) $\omega_{L} / w=3.0$.

nonzero magnetic fields, but for reasons of brevity they are not plotted.

With increasing magnetic field the oscillations in the density gradually become less pronounced and the confinement is enhanced as $\omega_{L}$ increases. The density profile changes whenever the magnetic susceptibility (which is proportional to $\partial E_{G} / \partial \omega_{L}$ with $E_{G}=\Sigma_{E<E_{F}} E$ the ground state energy) exhibits a discontinuity as a function of the magnetic field. For $\omega_{L} \gg w$ the energy spectrum behaves like a Landau spectrum. This is illustrated for the noninteracting case $(w=\Omega)$ in Fig. 2(a) and Fig. 2(b) for $N=10$ electrons, and in Fig. 3(a) and Fig. 3(b) for $N=28$ electrons. The insets show the magnetic susceptibility as a function of $\omega_{L}$.

\section{B. Density in the $x z$ plane}

Like the density in the $x y$ plane, the density profile in the $x z$ plane changes whenever there is a jump in the magnetic susceptibility. Figure 4 shows density contour plots for four fermions $(w=\Omega)$ for various magnetic fields. The asymmetry of the $x$ and $z$ directions due to the magnetic field along the $z$ axis is clearly revealed in the plots. In Fig. 5 the density contours for ten fermions $(w=\Omega)$ in the $x z$ plane are illustrated.

\section{CONCLUSION AND DISCUSSION}

In this paper we have presented analytical results for the density of spin-polarized harmonically interacting fermion oscillators in a magnetic field, taking the fermion statistics of the particles into account. The approach presented here is valid for any number of electrons and for any temperature. We concentrated on the ground state density for a number of 

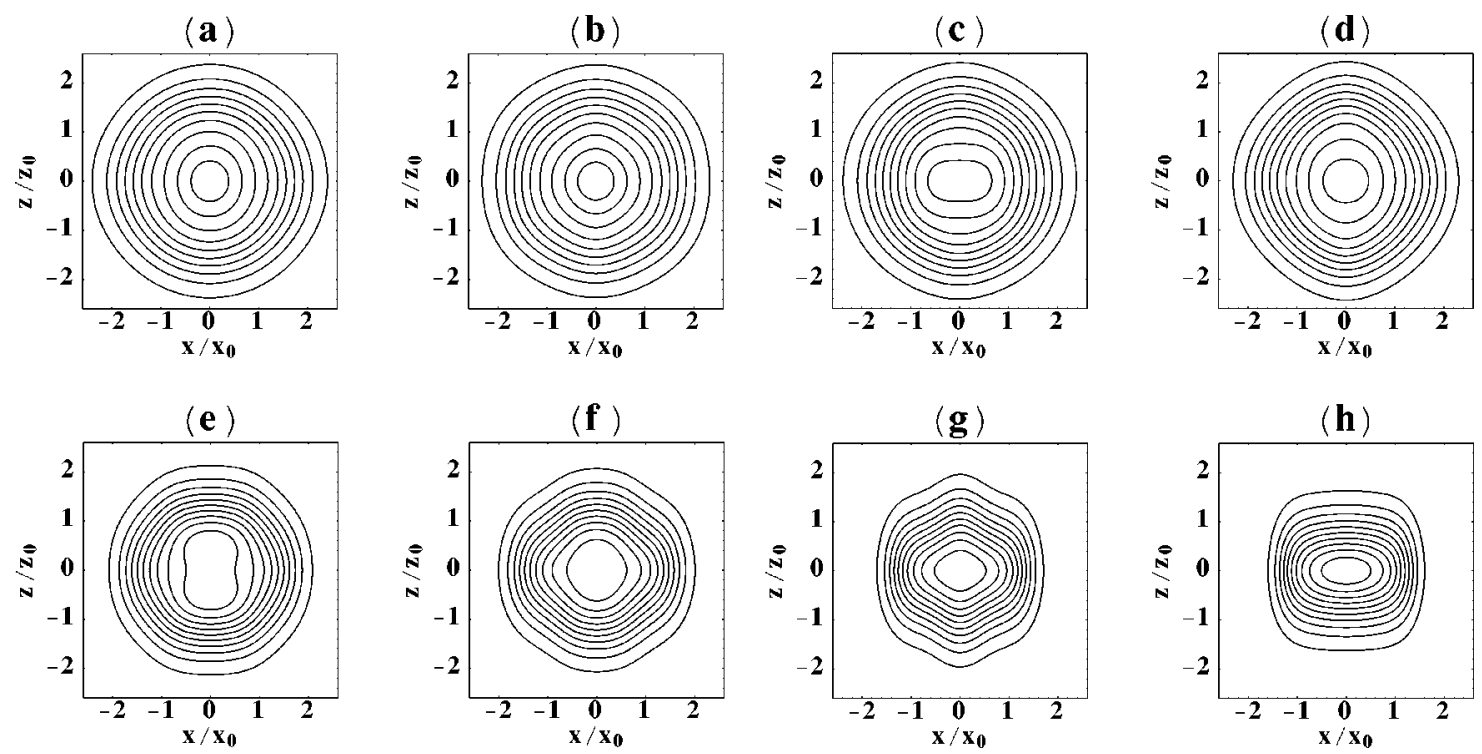

FIG. 5. Contour plots of $N n(x, z) / w$ for $N=10$ fermions and for $\Omega=w$ as a function of the scaled coordinates $x / x_{0}$ and $z / z_{0}$ with $x_{0}$ $=z_{0}=1 / \sqrt{w}$, measured from the center of the parabolic confinement potential for different values of the magnetic field: (a) $\omega_{L} / w=0.3,(\mathrm{~b})$ $\omega_{L} / w=0.5$, (c) $\omega_{L} / w=0.7$, (d) $\omega_{L} / w=1.2$; (e) $\omega_{L} / w=2.0$, (f) $\omega_{L} / w=2.5$, (g) $\omega_{L} / w=4.0$, (h) $\omega_{L} / w=5.0$.

particles ranging from 1 up to 45, although higher particle numbers can also be treated. The density in the $x y$ as well as in the $x z$ plane shows a magnetic field dependency that is governed by the discontinuity in the magnetic susceptibility. Oscillations are present in the density and they are more pronounced as the number of particles is increased. These oscillations are smoothed out and finally disappear with increasing Larmor frequency $\omega_{L}$. Whenever the magnetic field causes a discontinuity in the magnetic susceptibility, the density profile undergoes a sudden change, thus providing a means for characterizing the parameters of the system. To the best of our knowledge, the path integral approach used in this paper is the only method so far that provides this detailed information on the density for an interacting fermion system.

\section{ACKNOWLEDGMENTS}

Part of this work was performed in the framework of FWO Project Nos. G.0287.95, 1.5.545.98, G.0071.98, and WO.073.94N [Wetenschappelijke Onderzoeksgemeenschap over "Laag-dimensionele systemen" (Scientific Research Community on Low-Dimensional Systems)], the "Interuniversitaire Attractiepolen-Belgische Staat, Diensten van de Eerste Minister-Wetenschappelijke, Technische en Culturele aangelegenheden" (Interuniversity Poles of Attraction Programs-Belgian State, Prime Minister's Office-Federal Office for Scientific, Technical and Cultural Affairs), and in the framework of the GOA BOF UA 2000 projects of the Universiteit Antwerpen. F.B. and S.F. acknowledge the FWO (Fonds voor Wetenschappelijk OnderzoekVlaanderen) for financial support.
[1] L. P. Kouwenhoven et al., in Proceedings of the Advanced Study Institute on Mesoscopic Electron Transport, edited by L. L. Sohn, L. P. Kouwenhoven, and G. Shön (Kluwer, Dordrecht, 1997).

[2] S. Tarucha, D. G. Austing, T. Honda, R. J. van der Hage, and L. P. Kouwenhoven, Phys. Rev. Lett. 77, 3631 (1996).

[3] V. Fock, Z. Phys. 47, 446 (1928); C. G. Darwin, Proc. Cambridge Philos. Soc. 27, 86 (1930).

[4] R. C. Ashoori, Nature (London) 379, 413 (1996).

[5] N. B. Zhitenev, R. C. Ashoori, L. N. Pfeiffer, and K. W. West, Phys. Rev. Lett. 79, 2308 (1997).

[6] S. Tarucha, D. G. Austing, Y. Tokura, W. G. van der Wiel, and L. P. Kouwenhoven, Phys. Rev. Lett. 84, 2485 (2000).

[7] M. Koskinen, M. Manninen, and S. M. Riemann, Phys. Rev. Lett. 79, 1389 (1997).

[8] H.-M. Müller and S. E. Koonin, Phys. Rev. B 54, 14532 (1996).
[9] I.-H. Lee, V. Rao, R. M. Martin, and J.-P. Leburton, Phys. Rev. B 57, 9035 (1998).

[10] N. F. Johnson and M. C. Payne, Phys. Rev. Lett. 67, 1157 (1991).

[11] A. Angelucci and A. Tagliacozzo, Phys. Rev. B 50, 7088 (1997).

[12] S. Foulon, F. Brosens, J. T. Devreese, and L. F. Lemmens, Phys. Rev. E 59, 3911 (1999).

[13] R. P. Feynman, Statistical Mechanics, a Set of Lectures (W. A. Benjamin Inc., Reading, MA, 1972).

[14] J. Tempere, F. Brosens, L. F. Lemmens, and J. T. Devreese, Phys. Rev. A 61, 043605 (2000).

[15] L. F. Lemmens, F. Brosens, and J. T. Devreese, Phys. Rev. E 61, 3358 (2000)

[16] R. P. Feynman and A. R. Hibbs, Quantum Mechanics and Path Integrals (McGraw Hill, New York, 1965). 
[17] F. Brosens, J. T. Devreese, and L. F. Lemmens, Phys. Rev. E 55, 227 (1997); 55, 6795 (1997).

[18] F. Brosens, J. T. Devreese, and L. F. Lemmens, Phys. Rev. E 57, 3871 (1998); 58, 1634 (1998).

[19] L. F. Lemmens, F. Brosens, and J. T. Devreese, Solid State Commun. 109, 615 (1999).
[20] B. Simon, Functional Integration and Quantum Physics (Academic, New York, 1979).

[21] L. S. Schulman, Techniques and Applications of Path Integration (Wiley, New York, 1979).

[22] J. Tempere, F. Brosens, L. F. Lemmens, and J. T. Devreese, Solid State Commun. 110, 197 (1999). 